\title{
Intracoronary mitochondrial transplantation
}

a unique
opportunity
to preserve
or restore
myocardial
function via
metabolic
modulation
with infusion
of intact
organelles

In a paper published in JACC: Basic to Translational Science, James McCully and colleagues report the latest in a series of experiments on autologous mitochondrial transplantation for myocardial protection. Their findings indicate that mitochondrial transplantation into the heart is feasible, safe and beneficial; however, the mechanisms by which these benefits are achieved are uncertain.

McCully and co-workers have previously used autologous mitochondria isolated from skeletal muscle injected directly into the myocardium in both experimental animals and five paediatric patients with myocardial ischaemia. The investigators reported that transplanted mitochondria were internalized into cardiac cells, which led to increased myocardial ATP production and improved tissue viability and contractile function of injured myocardium. Despite these benefits, this approach is limited by the need for openheart surgery, multiple injections and physical manipulation of the heart to distribute the mitochondria. Therefore, the researchers aimed to investigate the biodistribution,

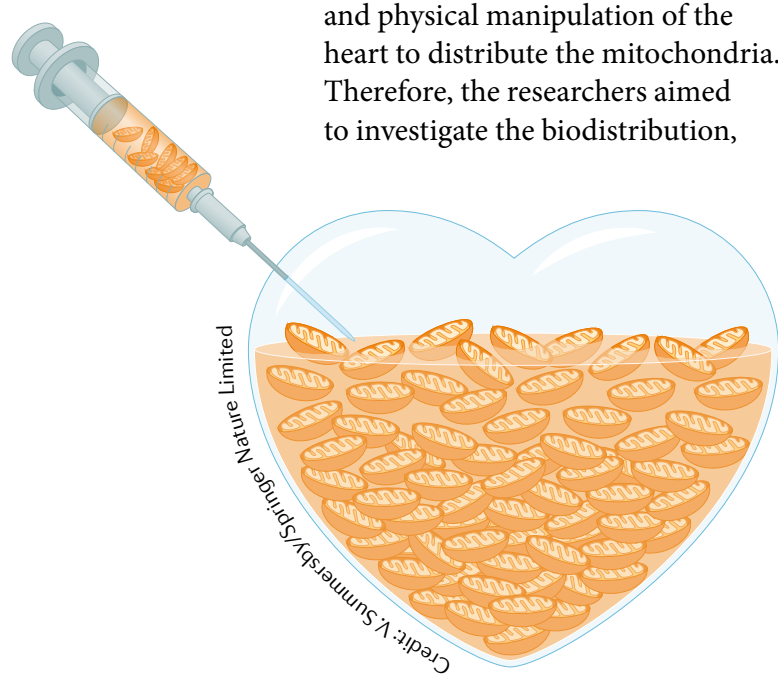

safety and efficacy of intracoronary delivery of mitochondria in pigs.

Intracoronary injection of radiolabelled mitochondria in the left coronary artery of healthy pigs resulted in transplanted mitochondria specifically in the left ventricle, with little or no tracer detected in any other organs, and without negative haemodynamic effects. Higher concentrations of mitochondria were associated with significant improvements in regional and global left ventricular function. Moreover, intracoronary injection of mitochondria increased coronary blood flow in a concentration-dependent manner. The researchers suggest that this effect was dependent on intact mitochondrial viability, ATP production and, in part, the activation of inwardly rectifying $\mathrm{K}^{+}$channels in blood vessels.

Next, intracoronary mitochondrial delivery was performed in pigs subjected to regional ischaemia-reperfusion injury. Pigs with mitochondrial transplantation had improved myocardial function, increased coronary blood flow and reduced infarct size compared with vehicle-injected animals.

"Intracoronary delivery of mitochondria to the myocardium provides a variety of possible applications for both percutaneous and surgical therapies by exploiting its multifactorial rescue of myocellular damage, coronary vasodilatation, and the versatility of endovascular catheter-based delivery," concludes McCully. In an editorial comment accompanying the article, W. H. Wilson Tang concurs that the "prospects of autologous mitochondrial transplantation will likely be disruptive. It may create a unique opportunity to preserve or restore myocardial function via metabolic modulation with infusion of intact organelles." However, he warns that "the lack of blinding of the measurements and the lack of randomization may have limited the confidence of the findings".

Christoph Maack (University Clinic, Würzburg, Germany), who was not involved in the study, cautions that the mechanisms of benefit must be investigated. Writing in The Journal of Clinical Investigation with Edoardo Bertero and Brian O'Rourke, Maack comments that mitochondria are unlikely to survive in a high $\mathrm{Ca}^{2+}$ extracellular milieu and remain viable. In addition, they state that the mechanism by which mitochondria might enter cardiomyocytes in vivo is unproven and, moreover, the number of mitochondria internalized into cardiomyocytes is likely to be too few to produce a sufficient increase in ATP levels to have a meaningful effect on cardiac function. Maack and colleagues emphasize the importance of "further clarifying the mechanisms that underlie the cardioprotective effects before this procedure is further applied in humans".

Gregory B. Lim

ORIGINAL ARTICLE Shin, B. et al. A novel biological strategy for myocardial protection by intracoronary delivery of mitochondria: safety and efficacy.JACC Basic Transl Sci. 4, 871-888 (2019)

RELATED ARTICLES Bertero, E. et al. Mitochondrial transplantation in humans: "magical" cure or cause for concern? J. Clin. Invest. 128, 5191-5194 (2018) |Bonora, M. et al. Targeting mitochondria for cardiovascular disorders: therapeutic potential and obstacles. Nat. Rev. Cardiol. 16, 33-55 (2019) 